\title{
"Can You Cure me? Children With Autism Spectrum Disorders Playing a Doctor Game With a Social Robot"
}

\author{
Ramona Simut, ${ }^{1}$ Cristina A. Costescu, $2,{ }^{2}$ Johan Vanderfaeillie, ${ }^{1}$ Greet van de Perre, ${ }^{3}$ Bram \\ Vanderborght, ${ }^{3}$ and Dirk Lefeber ${ }^{3}$ \\ ${ }^{1}$ Clinical and Life Span Psychology Department, Vrije Universiteit Brussel, Brussel, Belgium \\ ${ }^{2}$ Department of Clinical Psychology and Psychotherapy, Babes-Bolyai University, Cluj, Romania \\ ${ }^{3}$ Multibody Mechanics and Robotics Research Group, Vrije Universiteit Brussel, Brussel, Belgium \\ "Corresponding author: Cristina A. Costescu, Department of Clinical Psychology and Psychotherapy, Babes-Bolyai University, Cluj, Romania. Tel: +40-742213337, E-mail: \\ christina.costescu@gmail.com
}

Received 2015 May 25; Revised 2015 August 20; Accepted 2015 October 05.

\begin{abstract}
Background: Social robots may represent an important technological instrument for improving the social abilities of children with autism spectrum disorders (ASD).

Objectives: This work describes exploratory research in which a doctor game scenario, involving a social robot and a human partner, was implemented and tested with children with ASD.

Patients and Methods: Thirty children between the ages of four and eight who had been diagnosed with ASD were included in this study. The children alternated between playing a doctor game with the social robot Probo and playing the same game with a human partner, and were exposed to each condition only once in a counter-balanced order. The symbolic play task and the experimental setup were designed to test whether the children would engage in more collaborative behaviors while playing the doctor game with a social robot than performing the same activities with the human partner.

Results: Although the children with ASD were more interested in and entertained by the robotic partner, the children did not have better performance in the game, and did not show significantly more collaborative play and engagement while playing with the social robot, compared with the human partner.

Conclusions: A longer and more thorough study is needed to interpret these findings and better understand the ASD child-robot interaction. This paper concludes with a discussion of the related literature and proposals for future research directions.
\end{abstract}

Keywords: Robot-Assisted Therapy, Autism Spectrum Disorders, Social Robot, Symbolic Play, Collaborative Play

\section{Background}

Delays or abnormal functioning in symbolic play are among the most prominent characteristics of children on the autism spectrum. Previous studies have found that children with autism engage in spontaneous pretend play less frequently than other children, and with behaviors that are repetitive, stereotypic, and with less novelty and variety $(1,2)$. It is well established that play experiences are significant for child development, socialization, and cultural participation (3). However, developmental and sociocultural factors place children with ASD at risk for being excluded from these essential experiences (4).

Research has shown that children with ASD may learn to engage in higher levels of play, such as symbolic play. Lewis and Boucher (5) found that symbolic play can be elicited both verbally and through modelling, despite the fact that a lack of social motivation in children with ASD makes those social and play skills some of the most challenging skills to teach (6). Although it is often claimed that this social motivation is impaired in autism, (7) several studies have hypothesized that children with ASD can successfully engage in social interactions if social information is presented in an attractive manner, that is, in a manner that is easily understood and clearly identifies the expected behaviors (8).

A growing number of studies focus on using social robots as motivational tools for autism interventions. Although there are not enough rigorous data to support a positive therapeutic effect of social robots for autism interventions, observations commonly made in studies in this field are: (1) children with ASD enjoy the interaction with the robot and are motivated to engage in the tasks when assisted by the robot; (2) few children manifest anxious behaviors related to the robot or need time to habituate to it, even if they are, in general, sensitive to novel stimuli exposure; (3) the robot is able to trigger desirable social behaviors for the majority of the children with autism, and for some of them, the robot elicits behaviors that they 
normally do not manifest or manifest less frequently in human-human interactions, such as joint-attention or positive affect behaviors; and (4) there are consistent data showing increased levels of attention, such as more eyecontact behaviors, with a robot compared with a human partner during interactions (9-13).

In the past few years there have been multiple examples of studies using robots to involve children with ASD in play activities, for either therapeutic or educational purposes (14-16). These have shown that children with ASD seem to be very engaged and motivated in games in which the playmate is a social robot, and that social robots can stimulate dyadic play, imitative play, and turn-taking skills in children with ASD (17-19). Clear scenarios that describe the best way to integrate robots into play sessions have not yet been developed. In general, the robot-assisted tasks described in the literature consist of free interactions, and during those interactions, children are allowed to explore the robot and its capabilities. Few of the existing studies chose a standardized interactive scenario in which each interaction partner (child, robot, and therapist) had a specific role described in a pre-established protocol $(10,20)$.

\section{Objectives}

The aim of this study was to explore how children with ASD manifest their play skills in a pre-established scenario for a doctor game (Figure 1). Our purpose was to test whether there are significant behavioral changes in children with ASD between interactions with a robot and interactions with a human. Specifically, we tested whether the presence of a social robot influences the performance and engagement in play of children with ASD, and whether a robot can increase the social interaction between the child and his interactional partner during a play task. This study will measure the play behaviors of children with ASD and will investigate the effects that the child-robot interaction has on the children with ASD behaviors (i.e. how social behaviors modify during the interaction).

We tested the following hypotheses: 1) children with ASD will perform better in the "playing doctor" task when interacting with a robot than when interacting with an adult. The assumption behind this hypothesis is that children with ASD find the robot more motivating, and will therefore be more attentive to the task; 2) Children with ASD will display more collaborative play and less independent play in interaction with the robot than with an adult. In the interaction with the robot, the child will engage more in the same activity, will share more, and will better fulfill the needs of the robot than in the interaction with an adult;3) Children with ASD will be more engaged in the task when interacting with the robot than with an adult.
Before the experiments took place in Flanders, a pilot study in Romania was developed (21).

\section{Patients and Methods}

\subsection{Participants}

Thirty-five children with ASD were recruited from three Belgian schools for children with special needs. In order to be included in the study, the participants met the following criteria: 1) a diagnosis of ASD according to the criteria outlined in the Diagnostic and statistical manual of mental disorders IV(22);2) age between 5 and 8 years (Table 1);3) IQ $\geq 70 ; 4$ ) the ability to use language for communication, that is, the ability to create three-word sentences, at a minimum; 5) no diagnoses for other disabilities aside from ASD; and 6) parental informed consent was required for each of the participants before the study took place. IQ scores were assessed with the Wechsler Preschool and Primary Scale of Intelligence-III (WPPSI-III-NL, (23), N = 23), and the SnijdersOomen non-verbal intelligence test revised version (SON-R $2_{1 / 2}-7,(24), \mathrm{N}=7$ ). The mean IQ score was 91.23 ( $\mathrm{min}=70$ and $\max =119)$. No drugs were provided to the participants. All parents had the right to stop their children's participation at any time during the experiment. Out of 35 participants, 30 (27 boys and 3 girls) fit the inclusion criteria and performed both of the experimental conditions. The required number of the participants was determined using the program $\mathrm{G}^{*}$ Power. A medium effect size of $\mathrm{d}=0.5$ was established, and fixed values used for statistical power (0.8) and the level of significance $(\alpha=0.05)$, which are values widelyaccepted by the scientific community.

\subsection{Setting}

The study took place in the three selected schools, in rooms at least $20 \mathrm{~m} 2$ in area. The room used in each school was divided by a partition. One half was used as the control room, where the operator controlled the robot using a set of buttons on the PC screen, in a wizard of Oz (WoZ) setup. AWoZ setup implies the presence of a person who remotely operates the robot, which can act anywhere along the autonomy spectrum, from fully autonomous to fully tele-operated, as well as with mixed-initiative interaction (25). The operator took care of the higher-level decisions, controlled the gaze, and made Probo show different emotions. The operator was able to see into the therapy room through a video camera inside the robot's head, which provided a general view of the setting. The other half of the room included the interaction partner (the adult or the robot) with the participant, always accompanied by the experimenter. Two pillows were placed in front of the robot, one pillow for the experimenter and one for the child. The 

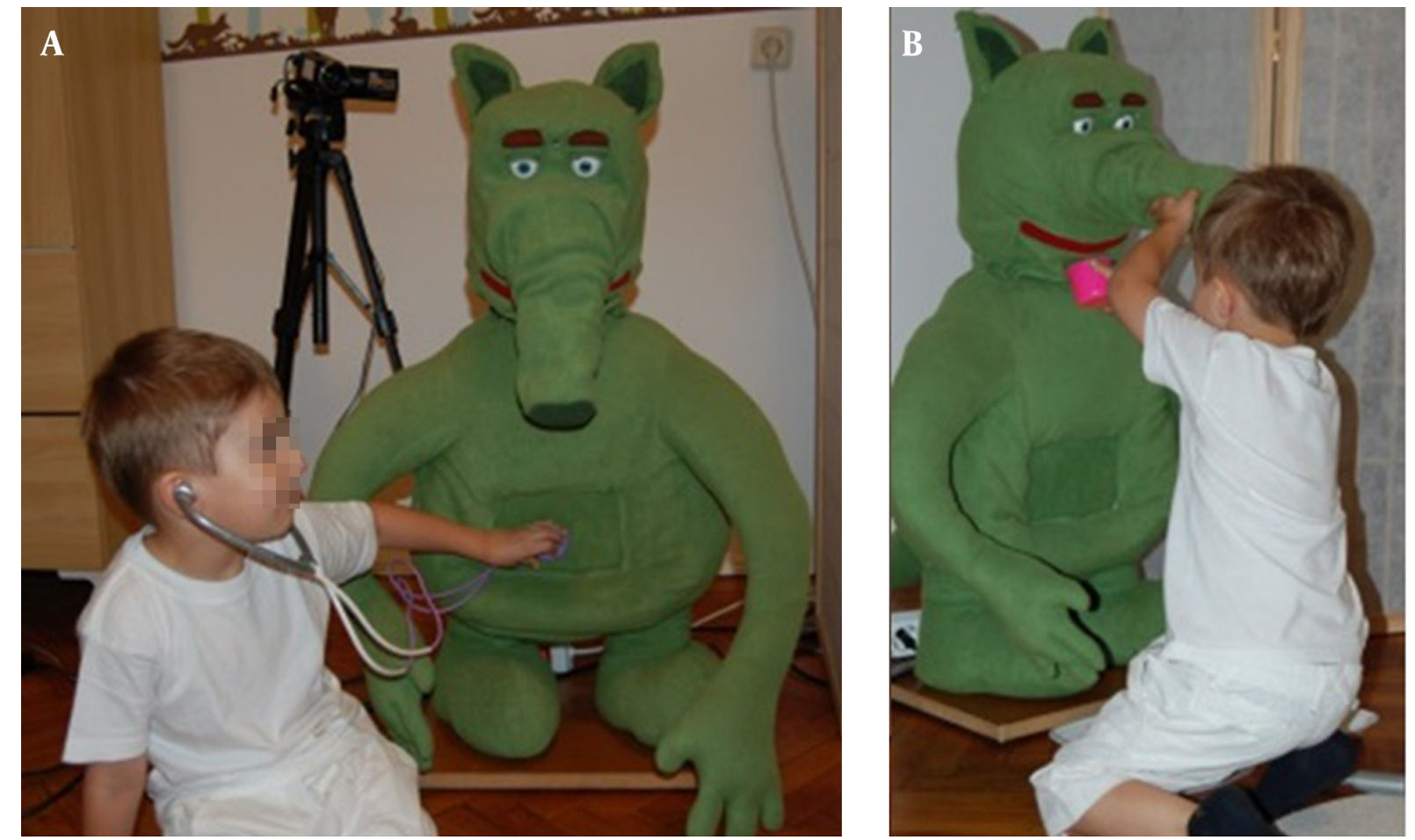

Figure 1. Child With ASD Playing a Doctor Game With the Social Robot Probo

Table 1. Age of the 30 Children Included in the Study

\begin{tabular}{llccc}
\hline & N & Minimum & Maximum & Mean \pm SD \\
\hline Age & 30 & 5 & 8 & $6.67 \pm 0.92$ \\
\hline
\end{tabular}

boxes with all the objects needed for activities were placed between the interaction partner and the child. Two digital cameras were used to record the interaction; a frontal camera recorded the child's upper torso and face, and a lateral one captured the child, the interaction partner, and the experimenter (Figure 2).

\subsection{Experimental Design}

A repeated measurements design was used in order to compare how children in the same group interacted with the two interaction partners and to determine whether children behave differently from one interaction to another. Each of the 30 participants was therefore exposed randomly to two conditions: the adult condition (AC), where the interaction partner was a human agent, and the robot condition (RC), in which they interacted with the social robot Probo. The interval between tests under the two conditions was between seven and ten days for each participant in the study. Sixteen of the children started with the robot condition, and the other 14 started with the adult condition. The same experimental task was established for both of the conditions, and the two interaction partners were trained to manifest the same verbal and non-verbal behaviors. The only difference between the two conditions was the type of agent, that is, the adult or the social robot Probo. A program created by the engineer of the team created a simple randomization sequence such that 16 children started with the robot condition and ended with the adult condition, while the other 14 started with the adult condition and ended with the robot condition.

\subsection{Instruments}

The social robot Probo was used in this study. To avoid expectations in behavior, the appearance of Probo does not resemble an existing human or animal, but an imaginary entity (Figure 3) (26). Probo's purpose is to serve as a multidisciplinary research platform for human-robot interaction and development of robot-assisted therapies focused 
Figure 2. Screenshot From Each of the Two Conditions of the Study Showing Child, Experimenter, and Interaction Partner
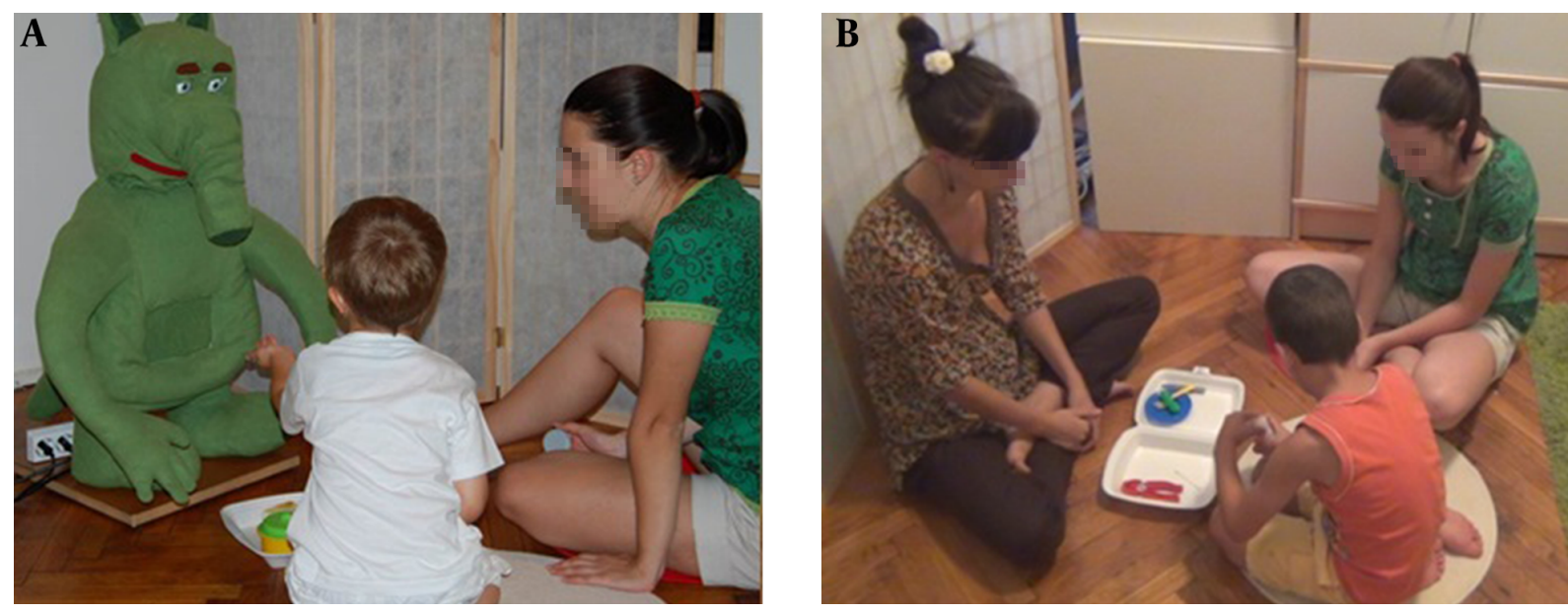

A, robot; B, an adult partner.

on children. The robot is designed to act as a social interface by employing human-like social cues and communication modalities. With 20 motors in the head, the robot is able to direct its gaze (eyes and head), is able to express emotions, animations, and facial expressions, and is capable of verbal communication (27). A lip-synch module allows the lips to move according to the voice (26).

To guarantee safe physical interactions between the robot and the child, and to provide a soft touch and huggability, the robot uses compliant actuation systems and a structure layered with foam and fabric. A user-friendly robot control center enables the operator to control the robot in an intuitive way in a WoZ setting. To facilitate a more fluent interaction and rigorous scenario execution, specific buttons for each animation were created according to the order needed for the scenario (Figure 4).

\subsection{Procedure}

Each of the 30 participants was exposed to both of the conditions; 16 children started with the robot condition and ended with the adult condition, and the other 14 started with the adult condition and ended with the robot condition. The experimenter conducted the task, following a pre-established scenario. The experimental task involved a single exposure for a maximum 15 minutes per child, with two demos and six trials. In the play task, a set of four objects (one neutral and three from the doctor set) were placed before the child, and the robot and the experimenter guided the game based on a standard protocol (accessible by writing to the first author). The robot or the adult was programmed or trained to tell the child his needs, and the child was asked to fulfill them by playing with the objects. The child was instructed to use the objects in the play task according to the needs of the robot or adult. After the interaction partner stated his needs, the child received a box with four objects, from which he/she was to choose the object that matched the need of the adult or robot. Table 2 gives a list of the objects in the play task.

Table 2. List of Objects Used in the Experimental Task

\begin{tabular}{|c|c|}
\hline Neutral Objects in the Box & $\begin{array}{l}\text { Doctor Set Object to be Used by } \\
\text { the Child }\end{array}$ \\
\hline $\begin{array}{l}\text { Shampoo, pills, plate with spoon, } \\
\text { pliers }\end{array}$ & pills \\
\hline $\begin{array}{l}\text { Spoon, toothpaste, stethoscope, } \\
\text { napkin }\end{array}$ & napkin \\
\hline $\begin{array}{l}\text { Plate, hammer, thermometer, } \\
\text { light }\end{array}$ & thermometer \\
\hline Pliers, fork, syrup, bandages & syrup \\
\hline $\begin{array}{l}\text { Knife, screwdriver, stethoscope, } \\
\text { thermometer }\end{array}$ & stethoscope \\
\hline Spoon, brush, syringe, tissue & syringe \\
\hline $\begin{array}{l}\text { Toothbrush, cup, thermometer, } \\
\text { bandages }\end{array}$ & bandages \\
\hline Tomato, pills, cup & cup \\
\hline
\end{tabular}

\subsection{Response Measurements}

We measured the performance of play, engagement in play, and the play style (collaborative vs. independent). The following list gives a detailed description of the measured variables: 

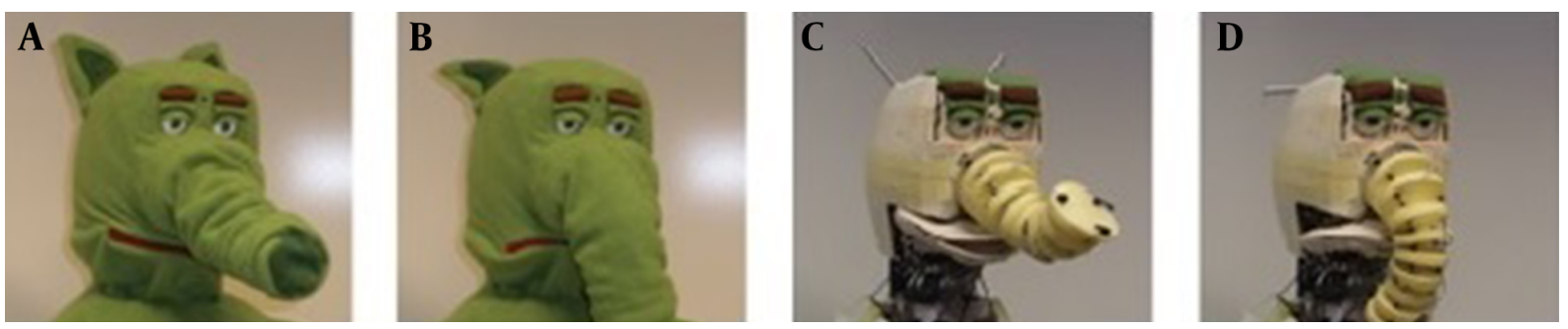

A and B, with cover; $C$ and $D$, without cover.

Figure 4. Robot Control Center (RCC) is Used to Control Probo in an Intuitive and Scenario-Specific Way

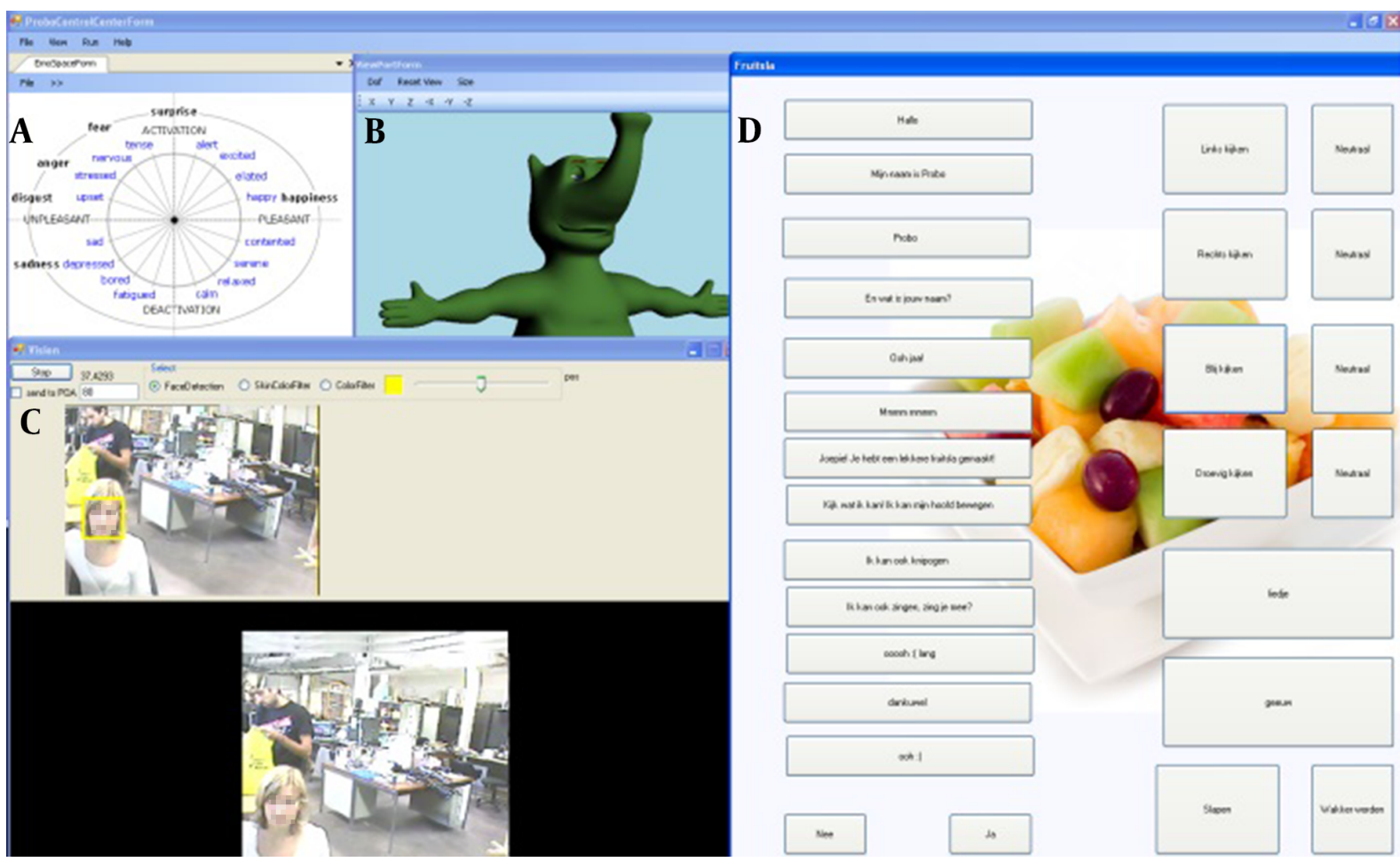

A, expressed emotion depicted in the 2dof emotion circumplex model of affect defined by Russell (Russell, 1980); B, the virtual 3D model of Probo used to visualize the motion behavior; C, video image and mosaic image constructed to obtain a full area view, covering the full visual range of the robot; D, the required buttons and their ordering, needed for following the requested protocol.

1) Play performance measured in frequency. More specifically, play performance was described by in how many opportunities of the play task, and whether the participant was playing appropriately or not, that is, using the correct objects in the play task.

2) Play style measured in duration (for example, how long the child used independent play) and in frequency (for example, how many actions were performed by the child with the object independently). Play style refers to the degree of independence and collaboration in play. Independent play was defined as play in which the child played alone and pursued activities independent of what the interactional partner was saying or doing. During collaborative play, the child played together with the interactional partner and directed his/her actions in response to the partner. The child and partner were engaged in the same activity, shared objects, and acted in order to fulfill the other's needs. To detect which play styles a child used, 
the duration and frequency of independent or collaborative actions were measured.

3) Engagement in play measured with the following variables:

3.1) Eye-contact behaviors, measured by their frequency and direction (to the interactional partner or to the experimenter). Eye contact was defined as looking at the face (or the upper region) of the experimenter or interactional partner, for example, when the child looked in the eye region of the interactional partner.

3.2) Social behaviors, measured with the following variables:

3.2.1) Escapes from the task, measured by their frequency. Escaping from the task was defined as every behavior of the child that indicated the child was not interested in the task, for example, when the child stood up and went to another place in the room.

3.2.2) Non-responses, measured by their frequency. A non-response was defined as a lack of response, either verbal or non-verbal, to a question or statement made by the experimenter, adult, or Probo (according to the protocol), within three seconds.

3.2.3) Positive affect measured in frequency. Positive affect was defined as smiling or laughing during the play task.

3.3) The verbal behaviors contingency, measured in frequency and direction to the interactional partner. This verbal utterance was defined as a verbal production that either expressed a complete proposition (subject + predicate) or was followed by more than 2 seconds of silence. The words that the child said during the play activity should be contingent in content with what the interactional partner (robot or adult) was saying (for example, in the doctor play, the child expressed that he will take the temperature) and also contingent in time (for example, the child had to express the verbal utterance within a maximum of 3 seconds after the last verbal behavior of the interactional partner).

All variables were manually coded with the program Elan-Linguistic Annotator, version 4.5.0. (27). This program offers the possibility to code not only the presence of the behavior (in this study, eye-contact and other behaviors), but also the exact time when the behavior occurred in the interaction. Using this data, it was possible for the first author to control and check the coding work of the two coders. Variables were only assessed during the task and not during the introduction or demonstration phases. The frontally-recorded films, capturing the face and upper torso of the child, were used for the coding process. Two master's students independently coded the recordings of the two conditions. The coders were blind to the experimental conditions. They were trained by the first author in data collection procedure. The training consisted in giving clear definitions of the dependent variables, in offering examples and non-examples for each category of behavior, and in observing appropriate behavior of normally-developing peers. Training continued until the inter-observer agreement reached $80 \%$ on two successive observations. An inter-observer agreement was calculated for $60 \%$ of the recordings, with a Cohen's kappa $>$ 0.74 for each of the variables.

\section{Results}

All data were analyzed using SPSS version 20. Firstly, a Mann-Whitney U test was used to investigate whether or not the order of the two conditions (robot interaction and adult interaction) had an impact on child performance. As described above, 16 children started with Probo interaction (Robot-Adult group) and 14 started with the adult condition (Adult-Robot group). Results showed no order effect for the majority of the variables, meaning that performance did not vary as a function of the order of the experimental conditions. A significant difference was observed for the solitary play with the $\operatorname{robot}(\mathrm{U}=64.50, \mathrm{z}=-2.597, \mathrm{P}$ $<0.05$ ) with a medium effect size $r=-0.47$, in favor of the Adult-Robot group.

The Wilcoxon signed-rank test was used to analyze the differences between the children's performance in the two conditions, adult (AC) and robot (RC). No significant differences were observed between the two conditions of the study for any of the variables (Table 3).

The Wilcoxon signed-rank test was also performed separately for the solitary play scenario (for which the order of the conditions proved to have an effect), in order to detect whether or not there was a significant difference between the adult and robot conditions in each of the two groups (Adult-Robot and Robot-Adult). No significant difference was found for solitary play between the two conditions of each of the groups (Table 4).

\section{Discussion}

Results showed that none of the hypotheses of this research could be confirmed. Our first hypothesis was that children will have better performance in a "playing doctor" task when interacting with the robot than with an adult. The fact that most of the children performed well in both of the conditions could be a possible explanation for not finding a significant difference in performances between conditions. Also, the mental age of the participants meant that for some of the children, the task was not challenging enough. 
Table 3. The Wilcoxon Signed-Ranks Test Values for Each of the Study Variables Between the Two Conditions

\begin{tabular}{|c|c|c|c|c|c|c|c|c|c|}
\hline & & PP & PS-C & EC-E & EC-P & VU & PA & NR & ET \\
\hline $\mathbf{Z}$ & & -0.35 & -0.70 & -1.58 & -0.27 & -0.31 & -0.01 & -1.00 & -0.81 \\
\hline \multicolumn{10}{|c|}{ RC } \\
\hline & Mean \pm SD & $4.87 \pm 1.77$ & $5.47 \pm 2.05$ & $13.40 \pm 9.56$ & $38.17 \pm 34.44$ & $17.73 \pm 17.84$ & $6.00 \pm 7.29$ & $0.00 \pm 0.00$ & $0.07 \pm 0.25$ \\
\hline & Mdn & 6.00 & 5.21 & 11.50 & 22.50 & 12.00 & 3.50 & 0.00 & 0.00 \\
\hline & Min & .00 & 3.38 & .00 & 5.00 & 0.00 & 0.00 & 0.00 & 0.00 \\
\hline & Max & 6.00 & 12.35 & 33.00 & 137.00 & 82.00 & 25.00 & 0.00 & 1.00 \\
\hline \multicolumn{10}{|c|}{ AC } \\
\hline & Mean \pm SD & $5.00 \pm 1.78$ & $5.39 \pm 2.16$ & $10.10 \pm 8.41$ & $33.27 \pm 19.06$ & $17.40 \pm 14.26$ & $6.07 \pm 6.16$ & $0.03 \pm 0.18$ & $0.13 \pm 0.43$ \\
\hline & Mdn & 6.00 & 4.79 & 8.50 & 24.50 & 13.50 & 4.00 & 0.00 & 0.00 \\
\hline & Min & 0.00 & 3.45 & 0.00 & 5.00 & 0.00 & 0.00 & 0.00 & 0.00 \\
\hline & Max & 6.00 & 14.00 & 32.00 & 81.00 & 47.00 & 21.00 & 1.00 & 2.00 \\
\hline
\end{tabular}

Abbreviations: AC, adult condition; EC-E, eye contact with the experimenter; EC-P, eye contact with the partner; ET, escaping from the task; NR, non-responses; PA, positive affect; PP, play performance measured in frequency; PS-C: play style-collaborative style measured in duration; and measured by their frequency; RC, robot condition.

The second hypothesis, which predicted that children with ASD will display more collaborative play and less independent play in interaction with the robot than with an adult, also could not be confirmed. However, the fact that the robot triggered a similar frequency of independent and collaborative play behaviors with an adult interaction partner again suggests that children with ASD experience no difficulties in understanding the behaviors of the robot Probo and engaging in a robot-assisted task. This suggests that research should continue to explore the potential value of robot-assisted therapy for autism.

There was also no confirmation of the third hypothesis, which predicted that children with ASD will be more engaged in the task when interacting with the robot than with an adult. This result is inconsistent with the outcomes of previous studies that suggest that children with ASD are more interested and better engaged in tasks when assisted by robots.

There are some differences between the outcomes of this study and those of previous studies, which have shown, for example, that children with ASD have more verbal utterances in the presence of the robot, (9) or manifested eye gaze and smile/laughter behaviors in interaction with a robot more frequently than in interaction with a human partner (20). These differences can be explained by several facts: 1 ) The positive outcomes were mostly obtained from engineer-driven work, and when the studies were conducted by psychologists, in a more controlled manner, the same positive outcomes were not statistically significant; 2) The robot Probo presents technological limitations on its ability to trigger some of the behaviors assessed in this study; 3) The characteristics of the children with autism in the present study were different than the subjects of previous work that showed statisticallysignificant results. Another possible explanation for our results is the small sample size. Although to the best of our knowledge, our sample size was significantly larger than that in previous studies, it may still have been too small to reveal small effects.

There were also some methodological issues in our study that should be noted. For example, one of the limitations in this study was the single exposure of the participants in the two conditions. Therefore, in future longitudinal studies, it could be interesting to explore in more depth the differences obtained between the two conditions after several exposures.

The technical constraints imposed by the robot also represent an important limitation of the present study. The WoZ setup used in this study is not efficient, and requires an additional human operator dedicated to controlling the robot. However, future work will focus on going beyond WoZ setups and towards robots that can operate somewhat autonomously (while of course remaining under the supervision of the therapist using a remote), so that an operator is no longer required. Moreover, at present the robot used is only capable of showing facial expressions and moving its head, eyes, ears, neck, and mouth. Different results may be obtained when its communication expressivity is also supported by gestures, such as the ability to move its arms and body). More social behaviors could also be elicited if the robot was capable of reacting to social advances made by the children with gestures, such as 
Table 4. The Wilcoxon Values for the Solitary Play Variable, for Both of the Two Groups (Robot-Adult and Adult-Robot)

\begin{tabular}{|c|c|c|c|}
\hline & & $\mathbf{N}$ & $\mathbf{Z}$ \\
\hline Robot-Adult & & 16 & -1.00 \\
\hline \multicolumn{4}{|l|}{ RC } \\
\hline Mean \pm SD & $003 \pm 0.14$ & & \\
\hline Mdn & 0.00 & & \\
\hline Min & 0.00 & & \\
\hline Max & 0.06 & & \\
\hline \multicolumn{4}{|l|}{$\mathrm{AC}$} \\
\hline Mean \pm SD & $0.06 \pm 0.24$ & & \\
\hline Mdn & 0.00 & & \\
\hline Min & 0.00 & & \\
\hline Max & 1.00 & & \\
\hline Adult-Robot & & 14 & -0.44 \\
\hline \multicolumn{4}{|l|}{$\mathrm{RC}$} \\
\hline Mean \pm SD & $0.33 \pm 0.62$ & & \\
\hline Mdn & 0.00 & & \\
\hline Min & 0.00 & & \\
\hline Max & 2.06 & & \\
\hline \multicolumn{4}{|l|}{$\mathrm{AC}$} \\
\hline Mean \pm SD & $0.49 \pm 1.16$ & & \\
\hline Mdn & 0.00 & & \\
\hline Min & 0.00 & & \\
\hline Max & 4.19 & & \\
\hline
\end{tabular}

Abbreviations: AC, adult condition; RC, robot condition.

pointing gestures.

In future research it could be interesting to examine over the long term the behaviors of children with ASD in interaction with a social robot. However, some first insights about how a specific category of the ASD spectrum (see the inclusion criteria of the participants) perceive and interact with the robot Probo are provided by the present study. Our observations will be useful to robot designers and for a multidisciplinary-team approach to this field, both for accelerating research outcomes and for overcoming the difficulties in communication experienced in this type of collaborative research.

\section{Acknowledgments}

The authors thank the children and their families, and the three Belgian schools for the hospitality to perform the experiments. A special thanks to the master's student, Deb- bie Willockx, for her important contribution to the implementation of different parts of the study.

\section{Footnotes}

Financial Disclosure: The first author of this study is being supported by Agency for innovation by science and technology (IWT) strategic basic research grantproject No. 121591. Other authors are supported by the CNCS-Bucharest, Romania project PN-II-IDPCE-2011-3-0484 -Exploring Robot-assisted therapy for children with ASD and the EU-FP7 project DREAM (project nr 611391).

Authors' Contribution: All authors have contributed equally to the study.

\section{References}

1. Baron-Cohen S. Autism and symbolic play. Br J Dev Psychol. 1987;5(2):139-48.

2. Kasari C, Gulsrud A, Freeman S, Paparella T, Hellemann G. Longitudinal follow-up of children with autism receiving targeted interventions on joint attention and play. J Am Acad Child Adolesc Psychiatry. 2012;51(5):487-95. doi: 10.1016/j.jaac.2012.02.019. [PubMed: 22525955].

3. Brown SL. Play: How it shapes the brain, opens the imagination, and invigorates the soul. Penguin; 2009.

4. Wolfberg P, Bottema-Beutel K, DeWitt M. Including children with autism in social and imaginary play with typical peers: Integrated play groups model. Am J Play. 2012;5(1):55.

5. Lewis V, Boucher J. Spontaneous, instructed and elicited play in relatively able autistic children. Br J Dev Psychol. 1988;6(4):325-39.

6. Weiss MJ, Harris SL. Teaching social skills to people with autism. Behav Modif. 2001;25(5):785-802. [PubMed: 11573340].

7. Chevallier C, Kohls G, Troiani V, Brodkin ES, Schultz RT. The social motivation theory of autism. Trends Cogn Sci. 2012;16(4):231-9. doi: 10.1016/j.tics.2012.02.007. [PubMed: 22425667].

8. Quirmbach LM, Lincoln AJ, Feinberg-Gizzo MJ, Ingersoll BR, Andrews SM. Social stories: mechanisms of effectiveness in increasing game play skills in children diagnosed with autism spectrum disorder using a pretest posttest repeated measures randomized control group design. J Autism Dev Disord. 2009;39(2):299-321. doi: 10.1007/s10803008-0628-9. [PubMed: 18704672].

9. Goodrich MA, Colton M, Brinton B, Fujiki M, Atherton JA, Robinson L, et al. a Robot into an Autism Therapy Team. IEEE Computer Society; 2012

10. Kim ES, Berkovits LD, Bernier EP, Leyzberg D, Shic F, Paul R, et al. Social robots as embedded reinforcers of social behavior in children with autism. J Autism Dev Disord. 2013;43(5):1038-49. doi: 10.1007/s10803012-1645-2. [PubMed: 23111617].

11. Ricks DJ, Colton MB, editors. Trends and considerations in robotassisted autism therapy. Robotics and Automation (ICRA), 2010 IEEE International Conference on. 2010; IEEE; pp. 4354-9.

12. Scassellati B, Admoni $\mathrm{H}$, Mataric M. Robots for use in autism research Annu Rev Biomed Eng. 2012;14:275-94. doi: 10.1146/annurev-bioeng071811-150036. [PubMed: 22577778].

13. Vanderborght B, Simut R, Saldien J, Pop C, Rusu AS, Pintea S, et al. Using the social robot probo as a social story telling agent for children with ASD. Interact Stud. 2012;13(3):348-72. doi:10.1075/is.13.3.02van.

14. Ferrari E, Robins B, Dautenhahn K. Therapeutic and educational objectives in robot assisted play for children with autism. In Robot and Human Interactive Communication. The 18th IEEE International Symposium. . 
15. Wainer J, Ferrari E, Dautenhahn K, Robins B. The effectiveness of using a robotics class to foster collaboration among groups of children with autism in an exploratory study. Pers Ubiquitous Comput. 2010;14(5):445-55.

16. Diehl JJ, Schmitt LM, Villano M, Crowell CR. The Clinical Use of Robots for Individuals with Autism Spectrum Disorders: A Critical Review. Res Autism Spectr Disord. 2012;6(1):249-62. doi: 10.1016/j.rasd.2011.05.006. [PubMed: 22125579].

17. Robins B, Dautenhahn K, Te Boekhorst R, Billard A. Robotic assistants in therapy and education of children with autism: can a small humanoid robot help encourage social interaction skills?. Univers Access InfSoc. 2005;4(2):105-20.

18. Kozima H, Nakagawa C, Yasuda Y. Children-robot interaction: a pilot study in autism therapy. Progress Brain Res. 2007;164:385-400.

19. Warren ZE, Zheng Z, Swanson AR, Bekele E, Zhang L, Crittendon JA, et al. Can robotic interaction improve joint attention skills?.JAutism Dev Disord. 2015;45(11):3726-34.

20. Tapus A, Peca A, Aly A, Pop C, Jisa L, Pintea S, et al. Children with autism social engagement in interaction with Nao, an imitative robot-A series of single case experiments. Interact Stud. 2012;13(3):315-47.
21. Pop CA, Pintea S, Vanderborght B, David DO. Enhancing play skills, engagement and social skills in a play task in ASD children by using robot-based interventions. A pilot study. Interact Stud. 2014;15(2):292320.

22. American Psychiatric Association . Diagnostic and statistical manual of mental disorders. ; 2003.

23. Hendriksen JGM, Hurks PPM. WPPSI-III-NL Wechsler Preschool and Primary Scale of Intelligence; Nederlandse bewerking. Maastricht University; 2009.

24. Tellegen PJ, Winkel M, Wijnberg-Williams BJ, Laros JA. Accountability and manual. Snijders-Oomen Nonverbal Intelligence Test; 1998.

25. Riek L. Wizard of Oz Studies in HRI: A Systematic Review and New Reporting Guidelines. J Human Robot Interact. 2012:119-36. doi: 10.5898/JHRI.1.1.Riek.

26. Saldien J, Goris K, Yilmazyildiz S, Verhelst W, Lefeber D. On the design of the huggable robot Probo. J Phys Agents. 2008;2(2):3-12.

27. Goris K, Saldien J, Vanderborght B, Lefeber D. How to achieve the huggable behavior of the social robot Probo? A reflection on the actuators. Mechatronics. 2011;21(3):490-500. 